\section{Distribution of Newly Synthesized Deoxyribonucleic Acid in Mitotic Division}

Firket and Verly recently presented ${ }^{1}$ some results on the labelling of chromosomal deoxyribonucleic acid in chick fibroblast cells with tritiated thymidine. One of their conclusions is that their results support the hypothesis of the equal distribution of labelled, and hence newly synthesized, deoxyribonucleic acid between sister anaphase and telophase chromosome sets and contradict an earlier observation by Mazia and $\mathrm{me}^{2}$ that this distribution may be decidedly unequal.

An inequality which exceeds that to be expected from the random distribution of the products of replication of different chromosomes and from the random distribution of decaying isotopic atoms suggests that there is an additional variant: a daughter chromosome may differ from its sister in the amount of newly synthesized deoxyribonucleic acid; in other words, the formation of daughter chromosomes from the maternal complex involves the random assortment of a small number of new and old units of deoxyribonucleic acid. This was the conclusion reached by us from an analysis which involved a mitotic system of six chromosomes and thymidine- ${ }^{14} \mathrm{C}$ as the deoxyribonucleic acid marker ${ }^{2}$.

A more direct approach to the problem was carried out by Taylor et al. ${ }^{3}$ : tritiated thymidine was sub. stituted for thymidine $-{ }^{14} \mathrm{C}$, thus permitting the autoradiographic analysis of single pairs of sister chromosomes rather than whole sets. Their conclusion was that sister chromosomes always shared equally in the deoxyribonucleic acid most recently synthesized, that the formation of daughter chromosomes did not involve the random assortment of the new and old structural units of the deoxyribonucleic acid of the mother complex. More recent experiments of mine strongly suggest, however, that these latter results are rather more complex than first appeared, that they are subject to a different interpretation from that offered by Taylor et al. ${ }^{3}$, and that they by no means exclude the earlier conclusion of random assortment. In short, the question of the mode by which the mother chromosome is transformed into its two daughters is still unresolved and in need of further experimental elucidation.

The study of Firket and Verly ${ }^{1}$ has a direct bearing on this question : if significant anaphase and telophase inequality of newly synthesized deoxyribonucleic acid is ruled out, then an equal partition of the material between daughter chromosomes is indicated. I should like to point out, however, that the experimental system chosen by these authors makes it virtually impossible to arrive at any significant quantitative conclusions. Unlike the experiment of Taylor et $a l .^{3}$, the study by Firket and Verly looks at whole sets of post-metaphase chromosomes. In this respect it resembles our earlier investigation of the six-chromosome system (Crepis capillaris). In sharp contrast with the latter, however, the chick fibroblast cell presents a very difficult statistical problem indeed: with upwards of 60 chromosomes the number of cells that must be analysed in order to separate the random inequalities introduced by the independent behaviour of different chromosomes from those which might be introduced by the segregation of chromosomal sub-units is extremely large. Similarly, the number of grains in the autoradiographic image should be large enough to permit the statistical separation of random distribution and decay errors from biologically significant inequality. The actual results on which Firket and Verly base their conclusion ${ }^{4}$ suggest that neither of these conditions is fulfilled. The number of cells analysed is 19, the average number of silver grains in the emulsion per cell is 47 . In such a sample of a mitotic distribution system with 60 or more chromosomes one cannot hope to detect inequalities which are ascribable to differential labelling of sister chromosomes. The only conclusion which can be drawn from these results is that newly synthesized deoxyribonucleic acid is distributed between post-metaphase chromosome sets in chick fibroblast cells. The question of the equality or inequality of this distribution remains unelucidated.

\section{W. Plaut}

Botany Department,

University of $W$ isconsin,

Madison 6, Wisconsin. April 17.

1 Firket, H., and Verly, W. G., Nature, 181, 274 (1957).

${ }^{2}$ Plaut, W., and Mazia, D., J. Biophys. and Biochem. Cytol., 2, 573 (1956). Taylor, J. H., Woods, P. S., and Hughes, W. L., Proc. U.S. Nat.
Acad. Sci., 43, 122 (1957).

${ }^{4}$ Firket, H., Arch. Biol., 69, 1 (1958).

I AGREE with Dr. Plaut that my results, though different from his, do not rule out the conclusions arrived at by him and Dr. Mazia. In fact, in the detailed paper ${ }^{1}$ to which he refers, I wrote (p. 84) : "Même si cette [Dr. Plaut and Mazia's] hypothèse sur la duplication de l'ADN est exacte, une telle déviation n'aurait qu'une chance infime d'apparaître dans les cellules de Poulet où le nombre de chromosomes est beaucoup plus élevé". I doubt the conclusions of their experiments for other reasons: namely, the inaccuracy of the limits of the field to be measured, the various possible errors in the quantitative assessment of radioactivity, and the contrary experiments by Taylor et al. ${ }^{2}$.

I quite agree, however, that no definite conclusions have yet been reached about the mode of distribution of new deoxyribonucleic acid among daughter chromosomes. It is both a difficult and important problem with far-reaching consequences. If a deoxyribonucleic acid unit is defined as a molecule or group of molecules that are synthesized and tied together, the possible hypotheses are as follows:

(a) The number of deoxyribonucleic acid units per chromosome is small and there is a mechanism that ensures a perfectly equal distribution of newly synthesized labelled units and old units among daughter chromosomes. This implies a definite spatial relationship between these units (or, less likely, a system that can distinguish between new and old units of identical composition).

(b) The number of units is small and they are randomly distributed among daughter chromosomes. On the average, two sister chromosomes have an equal amount of new and old deoxyribonucleic acid, but some pairs may show a very unequal distribution.

(c) The number of units per chromosome is large and there is a random distribution.

(d) The number of units is large but there is a tendency for the newly synthesized units to move together.

The experiments of Taylor et al. tend to prove hypothesis $(a)$. Plaut and Mazia's results ${ }^{3}$, taken at their face value, point to $(b)$ or $(d)$. Mine agree best with $(a)$ and $(c)$. It should also be borne in mind 